\title{
Intermediate acting non-depolarizing neuromuscular blocking agents and risk of postoperative respiratory complications: prospective propensity score matched cohort study
}

\begin{abstract}
Objective To determine whether use of intermediate acting neuromuscular blocking agents during general anesthesia increases the incidence of postoperative respiratory complications.

Design Prospective, propensity score matched cohort study.

Setting General teaching hospital in Boston, Massachusetts, United States, 2006-10.

Participants 18579 surgical patients who received intermediate acting neuromuscular blocking agents during surgery were matched by propensity score to 18579 reference patients who did not receive such agents.

Main outcome measures The main outcome measures were oxygen desaturation after extubation (hemoglobin oxygen saturation $<90 \%$ with a decrease in oxygen saturation after extubation of $>3 \%$ ) and reintubations requiring unplanned admission to an intensive care unit within seven days of surgery. We also evaluated effects on these outcome variables of qualitative monitoring of neuromuscular transmission (train-of-four ratio) and reversal of neuromuscular blockade with neostigmine to prevent residual postoperative neuromuscular blockade.
\end{abstract}

Results The use of intermediate acting neuromuscular blocking agents was associated with an increased risk of postoperative desaturation less than $90 \%$ after extubation (odds ratio $1.36,95 \%$ confidence interval 1.23 to 1.51 ) and reintubation requiring unplanned admission to an intensive care unit $(1.40,1.09$ to 1.80$)$. Qualitative monitoring of neuromuscular transmission did not decrease this risk and neostigmine reversal increased the risk of postoperative desaturation less than $90 \%$ (1.32, 1.20 to 1.46$)$ and reintubation $(1.76,1.38$ to 2.26$)$.

Conclusion The use of intermediate acting neuromuscular blocking agents during anesthesia was associated with an increased risk of clinically meaningful respiratory complications. Our data suggest that the strategies used in our trial to prevent residual postoperative neuromuscular blockade should be revisited.

\section{Introduction}

Tens of millions of people worldwide undergo general anesthesia every day. Anesthetists use a wide variety of drugs to establish the reversible state of anesthesia, which is characterised by hypnosis, amnesia, analgesia, hemodynamic stability with control of the stress response, and immobility. ${ }^{1}$ Deep anesthesia provides all components ${ }^{2}$; however, to achieve immobility with lower levels of anesthetic agents, anesthetists may coadminister curare-type neuromuscular blocking agents. These drugs inhibit neuromuscular transmission from nerves to muscles by competitively blocking the binding of acetylcholine to its postsynaptic receptors at the motor end plate, thereby causing paralysis of the muscle.

More than 400 million people receive neuromuscular blocking agents annually (Intercontinental Marketing Services (IMS) 
Health, Multinational Integrated Data Analysis System (MIDAS), September 2010), either in the operating theatre to optimize surgical conditions, or in the intensive care unit to facilitate mechanical ventilation in those with patient-ventilator asynchrony. ${ }^{4}$ Neuromuscular blocking agents are also commonly administered before tracheal intubation to ensure higher quality of the procedure and fewer complications. These drugs decrease intubation associated laryngeal morbidity as well as procedure related complications when used by experienced intensivists for emergency intubations. ${ }^{5-8}$

Full restoration of a patient's muscle strength is essential to ensure a safe postoperative recovery. Lingering effects of neuromuscular blocking agents, however, may cause partial paralysis, a condition in which symptoms of muscle weakness prevail in the postoperative period. ${ }^{9}$ This may impair breathing, upper airway patency, protective airway reflexes, swallowing, and coughing, thereby putting patients at risk for serious complications in the vulnerable postoperative period. ${ }^{10-15}$

Unanticipated postoperative intubation is associated with increased mortality and increasing healthcare costs. ${ }^{16}{ }^{17}$ The long acting neuromuscular blocking agent pancuronium has been associated with a higher risk of postoperative respiratory failure. ${ }^{18}{ }^{19}$ Consequently, long acting compounds have almost quantitatively disappeared from the market, being replaced by modern intermediate acting non-depolarizing neuromuscular blocking agents, but it is unclear if the use of these drugs represents a risk factor for adverse perioperative respiratory outcomes. $^{20}$

In addition to careful clinical assessment, anesthetists commonly use two strategies to control the effects of non-depolarizing neuromuscular blocking agents and restore patients' optimal muscle strength. Firstly, the monitoring of neuromuscular transmission is applied during surgery to assess the degree of a patient's neuromuscular block. Most often subjective (qualitative) visual or tactile (palpation) assessment of a muscle response to a train-of-four stimulation (series of four electric stimuli delivered at $2 \mathrm{~Hz}$ ) of a peripheral nerve is measured. After neuromuscular blocking agents have been administered, muscles show a decrease (fade) of muscular contraction from first to fourth response as opposed to muscles with intact or fully restored neuromuscular transmission, which show the same response to each stimulus. Secondly, reversal of neuromuscular blockade with acetylcholinesterase inhibitors is used to antagonise potentially lingering effects of non-depolarizing neuromuscular blocking agents at the end of surgery.

Acetylcholinesterase inhibitors like neostigmine increase the amount of acetylcholine in the synaptic cleft, thereby counteracting the effects of neuromuscular blocking agents. ${ }^{21}{ }^{22}$ Respiratory failure in the immediate postoperative period is related to a multiplicity of causes, such as the respiratory depressant effects of anesthetics, opioids, and neuromuscular blocking agents, the effects of the surgical trauma, and the consequences of bleeding and intraoperative hypothermia. Controlling for these variables to the best of our ability, we carried out a prospective propensity matched analysis. We hypothesized that the use of intermediate acting non-depolarizing neuromuscular blocking agents would be associated with an increased risk of postoperative hypoxic events and reintubation requiring unplanned admission to an intensive care unit and mechanical ventilation. We also explored whether the monitoring of neuromuscular transmission during surgery and reversal of neuromuscular blockade decrease the risk for postoperative respiratory adverse events.

\section{Methods}

Massachusetts General Hospital is a multidisciplinary, tertiary care facility and teaching affiliate of Harvard Medical School in Boston, Massachusetts, United States. More than 40000 surgical procedures (about 50\% each in inpatients and outpatients) are performed annually. We carried out a prospective analysis of filed data on all surgical patients who underwent general anesthesia at Massachusetts General Hospital from March 2006 to September 2010.

\section{Data sources and study population}

Three separate databases were utilized to integrate deidentified data from the preoperative, intraoperative, and postoperative period for patients who underwent a surgical procedure in the main operating suites. We included surgical procedures in which patients were tracheally intubated and ventilated for surgery and extubated at the end of surgery. We decided a priori to include all procedures that met the inclusion criteria rather than limiting the analysis to individual patients who underwent only one procedure; respiratory side effects of neuromuscular blocking agents are mainly procedure and dose specific, and inclusion of subsequent procedures of the same patient explains additional variance of the interplay between use of neuromuscular blocking agents and the occurrence of postoperative respiratory complications. The Anesthesia Information Management System was installed by the Department of Anesthesia, Critical Care and Pain Medicine in 2002. It records physiological data streaming from patient monitors as well as information on medical history and documentation of important surgery and anesthesia related events, including adverse events, perioperative procedures, and drug and fluid therapy. In addition we used the respiratory therapy quality assurance database and hospital billing database. Retrieved data on the patients' characteristics and preoperative medical data included sex, age, body mass index, American Society of Anesthesiologists physical status classification, and information on specific illnesses assembled in the Charlson comorbidity index. The last was calculated using codes of the International Statistical Classification of Diseases and Related Health Problems, ninth revision. Details of intraoperative management included surgical specialty (characterised as burn, gynecology, neurosurgery, urology, cardiac, general, oral/maxillofacial, orthopedic, pediatric, plastic, thoracic, transplant, trauma and vascular, and surgical oncology), duration of the surgical procedure, emergency status, monitoring of neuromuscular transmission, and the use of volatile anesthetics, nitrous oxide, opioids, and the reversal agent neostigmine.

\section{Neuromuscular blocking agents}

We screened all anesthetics to determine if neuromuscular blocking agents were administered during surgery. Agents that were administered comprised the depolarizing muscle relaxant succinylcholine, as well as non-depolarizing agents mivacurium (short acting), cisatracurium, rocuronium, vecuronium (all three intermediate acting), and pancuronium (long acting). In the statistical analysis we included all anesthetics in which intermediate acting non-depolarizing neuromuscular blocking agents were administered to patients intraoperatively. We also included in the analysis those anesthetics in which patients additionally or exclusively received succinylcholine for intubation. Ninety anesthetics were excluded for being associated with use of pancuronium (83 anesthetics) and mivacurium (seven anesthetics). Thirty two anesthetics were excluded because of missing points on core data. 


\section{Outcomes}

We chose desaturation after extubation and reintubation as the primary outcome measures and screened all the medical records for these two respiratory endpoints.

Desaturation after extubation was defined as hemoglobin oxygen saturation below $90 \%$ and below $80 \%$ during the first 20 minutes after extubation with a decrease in oxygen saturation after extubation of more than $3 \%$. Our electronic record of anesthesia (Metavision) samples data from a pulse oximeter every 20 seconds. For statistical analysis we used the nadir value taken during 10 epochs of one minute duration each before and after extubation; we included a desaturation event in the analysis if these criteria were fulfilled.

Reintubation was defined as any reintubation in the hospital after primary extubation in the operating room within seven days of surgery, requiring unplanned admission and ventilation in the intensive care unit. We excluded reintubations required for conducting a second surgical procedure.

For patients who underwent surgical procedures and were identified as being reintubated based on the combination of an extubation billing code followed by evidence of respiratory services afterwards, we determined the time between primary extubation and reintubation, as well as the proposed mechanism leading to reintubation. This was achieved by reviewing charts of all patients who presented with postoperative reintubation based on billing data.

We evaluated two secondary outcomes, which represent strategies used to prevent residual postoperative neuromuscular blockade: monitoring of neuromuscular transmission and reversal of neuromuscular blockade.

Monitoring of neuromuscular transmission was defined as any subjective, visual, or tactile assessment of the evoked response of the skeletal muscle to peripheral nerve stimulation.

Measurements are taken during surgery and entered electronically into the Anesthesia Information Management System by the anesthetist. The other secondary outcome was reversal of neuromuscular blockade, which was scored if neostigmine was administered during surgery and documented in the Anesthesia Information Management System. Additional secondary endpoints -included in the analysis were postoperative length of stay in hospital and in-hospital mortality. We retrieved both values from the hospital electronic medical record (discharge summary).

\section{Statistical analysis}

Statistical analysis was done using JMP (SAS Institute), SPSS (version 13.0), and SAS (version 9.2). We considered a two tailed $\mathrm{P}$ value of less than 0.05 as significant. To control for potential confounding, we used a propensity score matched cohort design.

\section{Propensity score}

Our data came from 46899 patients, 17\% of whom underwent more than one procedure. The propensity score for a patient is defined as the probability of being treated, conditional on pretreatment factors. To identify variables that went into the decision to treat a patient with a neuromuscular blocking agent, we polled anesthetists from our department to outline their decision making process on whether a surgical patient should or should not receive a neuromuscular blocking agent during surgery. We then selected covariates from our database to approximate this clinical approach and also included risk factors chosen a priori for the outcome events. More specifically, we considered age, sex, body weight, body mass index, American Society of Anesthesiologists physical status classification, surgical specialty, duration of the surgical procedure, emergency status, the Charlson comorbidity index, ${ }^{23}$ and use of volatile anesthetics, and nitrous oxide. These variables were then included in a multivariable logistic regression model, with intermediate acting non-depolarizing neuromuscular blocking agents administered intraoperatively as the dependent variable. We included missing information as indicator in the propensity score model. We reran the propensity score model and the matching procedure including information on whether a patient had undergone only one surgical procedure or more than one. This did not meaningfully change our findings.

\section{Matching}

On the basis of our calculated propensity scores, we matched people who had received at least one non-depolarizing neuromuscular blocking agent during surgery to those who did not receive any by using greedy matching. ${ }^{24}$ The greedy matching algorithm first identifies matched pairs (a treated person and an untreated person) within a closeness range of 0.00001 of the propensity score, then if no more individuals can be found, the program identifies matched pairs in a range of 0.0001 and so on up to a closeness range of 0.1 . We selected one reference surgical procedure for every patient exposed to non-depolarizing neuromuscular blocking agents, resulting in cohorts of 18579 surgical patients who received at least one non-depolarizing neuromuscular blocking agent and 18579 reference surgical patients. We then followed both cohorts for the occurrence of the outcome events. Using the propensity score as covariate in a regression outcome model in the entire study cohort resulted in similar effect estimates, indicating no strong differential effects among patients who could not be matched. ${ }^{25}$

\section{Outcome models}

Finally, we carried out logistic regression analysis to test the association between intermediate acting non-depolarizing neuromuscular blocking agents and the various outcomes in the matched cohort. To test the secondary research hypotheses that monitoring of neuromuscular transmission and reversal of neuromuscular blockade with neostigmine would influence the outcome criteria, we applied logistic regression analysis in the dataset of the propensity score matched cohort. Results are presented as odds ratios with $95 \%$ confidence intervals.

\section{Results}

Between 2006 and 2010 a total of 57068 surgical procedures were carried out under general anesthesia and intubation in 46 899 patients who met the inclusion criteria (fig $1 \Downarrow$ ). After propensity scores had been calculated, 18579 patients who received at least one intermediate acting non-depolarizing neuromuscular blocking agents were matched to 18579 reference patients who did not receive such agents.

\section{Total study population}

Table $1 \Downarrow$ shows the characteristics of patients who underwent surgical procedures in the total study population. Intermediate acting non-depolarizing neuromuscular blocking agents were administered to patients undergoing 32002 procedures in the following distribution: $66.2 \%$ for cisatracurium, $22.9 \%$ for vecuronium, and $11.6 \%$ for rocuronium. Only $50 \%$ of patients undergoing surgical procedures who received intermediate acting non-depolarizing neuromuscular blocking agents were reported 
to have been monitored for neuromuscular transmission, and a cholinesterase inhibitor (neostigmine) was administered in $63.6 \%$ of the patients who underwent surgery. Of the 32002 surgical procedures where patients received intermediate acting non-depolarizing neuromuscular blocking agents and neostigmine, $37.2 \%$ were also monitored for neuromuscular transmission.

\section{Propensity matched cohort}

Table $2 \Downarrow$ shows the characteristics of the propensity score matched cohorts. The baseline covariates were balanced between the treated and untreated groups. Cisatracurium was administered in $67.1 \%$ of surgical procedures, vecuronium in $22.4 \%$, and rocuronium in $11.1 \%$.

\section{Primary outcome: respiratory complications}

Of the 18579 patients who received at least one intermediate acting non-depolarizing neuromuscular blocking agent, 151 $(0.8 \%)$ were reintubated, $925(5.0 \%)$ had a desaturation event with hemoglobin oxygen saturation $<90 \%, 212(1.1 \%)$ had a desaturation event with hemoglobin oxygen saturation $<80 \%$, and $55(0.3 \%)$ died in hospital. The patients' hospital length of stay averaged 4.0 days. Logistic regression analysis showed that the use of intermediate acting non-depolarizing neuromuscular blocking agents was associated with an increased risk for both postoperative desaturation (oxygen saturation $<90 \%$, odds ratio $1.36,95 \%$ confidence interval 1.23 to 1.51 ; $\mathrm{P}<0.01$, oxygen saturation $<80 \%, 1.66,1.34$ to $2.07 ; \mathrm{P}<0.01$ ) and postoperative reintubation requiring admission to an intensive care unit $(1.40,1.09$ to $1.80 ; \mathrm{P}<0.01)$. When intermediate acting non-depolarizing neuromuscular blocking agents were administered for surgeries of short duration $(<120$ minutes), the risk of reintubation was even higher (2.04, 1.44 to $2.90 ; \mathrm{P}<0.01)$. Table $3 \Downarrow$ provides a summary of the associations between the use of intermediate acting non-depolarizing neuromuscular blocking agents and the different outcome variables.

Reintubation requiring unplanned admission to an intensive care unit is a serious complication, which translated to a more than 90-fold higher risk of dying in hospital (odds ratio 91, 67 to $125 ; \mathrm{P}<0.001)$. Most of the patients were intubated during the first three days after surgery, with the peak during the first 24 hours. Data for the entire study period and up to seven days after extubation was captured and is presented in fig $2 \Downarrow$, which shows the day of reintubation in patients where reintubation could clearly be allocated to a well defined time (fig 2). Fig $3 \Downarrow$ summarizes the mechanisms leading to respiratory failure and reintubation as determined by chart review. Specific information on the mechanisms leading to reintubation could be clearly identified in 216 out of 249 patients who were reintubated based on information derived from the billing code, 78 of whom were reintubated in the operating room and 138 out of the operating room (post-anesthesia care unit, intensive care unit, or on the surgical floor). Of those, the five most common mechanisms leading to reintubation (and subsequent unplanned admission to an intensive care unit) were postoperative pulmonary edema, pneumonia, atelectasis, impairment of brain function leading to sedation, and aspiration (fig 3 ). Clinicians provided multiple (on average 2.5 ) reasons why reintubation was required.

\section{Strategies to avoid residual postoperative neuromuscular blockade}

In the propensity score matched cohort, monitoring of neuromuscular transmission was only applied to half of the cases $(48.9 \%)$. The reversal agent neostigmine was administered in $63.2 \%$ of the patients, and $36.2 \%$ of those who received neostigmine were also monitored for neuromuscular transmission.

Use of the reversal agent neostigmine was associated with desaturation below $90 \%$ (odds ratio 1.32, 1.20 to 1.46), as well as reintubation requiring unplanned admission to an intensive care unit $(1.76,1.38$ to 2.26 , table $4 \Downarrow)$. Monitoring of neuromuscular transmission was also associated with increased risk of these two outcome events (table $4 \Downarrow$ ). However, in patients who received neostigmine for reversal, monitoring seemed to have some beneficial effects. In total, 9401patients who underwent surgical procedures received both neostigmine for reversal and monitoring of neuromuscular transmission. In this subgroup of cases, after extubation in the operating theatre, low level hypoxia (oxygen saturation $<90 \%$, and oxygen saturation $<93 \%$ ) occurred less often compared with those who received neostigmine without monitoring of neuromuscular transmission $(\mathrm{P}=0.003$ and $\mathrm{P}<0.001$, respectively).

\section{Discussion}

In this large, prospective propensity score matched cohort study, the use of intermediate acting non-depolarizing neuromuscular blocking agents was associated with adverse postoperative respiratory outcomes - that is, desaturation after extubation to a peripheral oxygen saturation of $<90 \%$ as well as $80 \%$, and postoperative reintubation requiring unplanned admission to an intensive care unit and mechanical ventilation. Qualitative monitoring of neuromuscular transmission (peripheral train-of-four nerve stimulation to visually screen for a fade of contraction) did not significantly modify the risk, and reversal with neostigmine even increased the risk of severe postoperative respiratory failure.

\section{Comparison to previous studies}

Several studies have reported adverse respiratory events after the use of neuromuscular blocking agents. The early finding that attracted the most attention and controversy was presented roughly 60 years ago when a six-fold higher mortality rate when neuromuscular blocking agents were added to anesthetics was reported. ${ }^{26}$ The authors could not adjust their findings for important variables affecting mortality, thereby promoting a potentially misleading relation. ${ }^{27}$ In the following 40 years, further outcome studies have been published to examine the association between the use of neuromuscular blocking agents and anesthesia related mortality. ${ }^{19} 262829$ One study used a prospective survey to study all mortality associated with anesthesia over a period of 10 years. ${ }^{28}$ The author reported that one of the main contributing factors to anesthesia related deaths was respiratory inadequacy after neuromuscular block. ${ }^{28}$ Other authors have reported on data collected by the Association of Anaesthetists of Great Britain and Ireland based on anonymous reports of deaths within six days of anesthesia. ${ }^{29}$ They found that reported anesthesia related deaths could mainly be attributed to postoperative respiratory failure, which was in many cases related to management of neuromuscular blocking agents. ${ }^{29}$ Researchers of a prospective, multicenter study reported that the long acting non-depolarizing neuromuscular blocking agent pancuronium was associated with a higher risk of postoperative pulmonary complications. ${ }^{19}$ Despite the introduction of intermediate acting neuromuscular blocking agents, more recent database studies published since the 1990s continue to show associations between the use of muscle relaxants as well as partial paralysis potentially related to neuromuscular 
management and adverse postoperative outcomes. ${ }^{30-32}$ Our findings extend those of these reports as we propose that intermediate acting non-depolarizing neuromuscular blocking agents are a risk factor for hypoxic events after extubation and increased reintubation rates. This is important, because intermediate acting non-depolarizing neuromuscular blocking agents now represent $80 \%$ of the market volume for neuromuscular blocking agents.

\section{Strategies to prevent postoperative residual neuromuscular blockade}

To help prevent these postoperative adverse effects of muscle relaxants, clinicians use two common strategies to avoid residual neuromuscular blockade and its consequences: intraoperative monitoring of neuromuscular transmission and pharmacological reversal of neuromuscular blockade. In our study, intraoperative qualitative monitoring of neuromuscular transmission (that is, visual or tactile identification of a fade of contraction after train-of-four stimulation of a peripheral nerve) was used by about $50 \%$ of the clinicians but did not help prevent postoperative respiratory complications associated with the use of neuromuscular blocking agents. In contrast, other researchers reported that quantitative intraoperative monitoring of neuromuscular transmission (that is, identification of a train-of-four fade through acceleromyography monitoring $)^{11}$ reduces the risk of residual neuromuscular blockade and adverse respiratory events in the post-anesthesia care unit. ${ }^{33}$

Acceleromyography monitoring uses a small piezoelectric transducer attached to the stimulated muscle to objectively measure the force produced by a muscle after nerve stimulation. While visual or tactile observation of fade may enable a clinician to predominantly identify distinct levels of partial paralysis, another study supports the view that quantitative monitoring of neuromuscular transmission may be required to adequately detect low but clinically meaningful levels of residual neuromuscular blockade. ${ }^{34}$

Pharmacological reversal of muscle relaxants by using acetylcholinesterase inhibitors in combination with glycopyrrolate at the end of the surgical procedure has been associated with a decreased risk of severe postoperative morbidity and mortality. ${ }^{31}$ In contrast, we found no beneficial, but rather a harmful, association of use of neostigmine on respiratory complications. Reversal of neuromuscular blockade at the end of surgery is important for patients' safety but it varies broadly between countries, medical centers, and anesthetists. The routine reversal of the effects of neuromuscular blocking agents at the end of every surgical procedure is rarely promoted in Europe, whereas antagonizing effects of non-depolarizing neuromuscular blocking agents is common practice in the United States, regardless of whether residual effects of the drugs can be shown. ${ }^{35}$ Routine reversal at the end of the surgical procedure has to be considered carefully and does not always ensure complete restoration of patients' muscle strength. On the contrary, neostigmine may not only cause a variety of side effects like arrhythmias, dry mouth, and bronchospasm but its use may have little influence on partial paralysis. ${ }^{36}$ In fact it has been shown to adversely affect neuromuscular function and cause muscle weakness in patients as well as healthy volunteers when given after complete recovery of neuromuscular transmission. ${ }^{35}{ }^{37-39}$ More specifically, when given in the absence of neuromuscular block, neostigmine can impair upper airway dilator volume, genioglossus muscle function, and diaphragmatic function, important factors to take into account with respiratory complications. ${ }^{21}{ }^{39}$ In our study we hypothesized that neostigmine induced neuromuscular block - that is, the manifestation of a depolarization block caused by accumulation of acetylcholine at the muscle endplate, may have occurred in those patients who received the acetylcholinesterase inhibitor as part of a "routine" reversal approach by the end of the surgical procedure without previous identification of a residual neuromuscular blockade by monitoring of neuromuscular transmission..$^{218-40}$ Other possible downsides of neostigmine reversal relate to its duration of action, which may be shorter than the elimination half life of rocuronium under specific conditions. In practical terms, the maximum depth of neuromuscular transmission block that can be sufficiently reversed by neostigmine approximately corresponds to the reappearance of the fourth muscle response to train-of-four stimulation. ${ }^{41}$ If neostigmine is given during deep neuromuscular block, absence of fade on subjective evaluation (such as palpation) of the response to train-of-four stimulation would mislead most clinicians into believing that adequate reversal had been achieved. In reference to a previous study, it is for this reason that antagonism with acetylcholinesterases is best delayed until at least two to three and preferably four responses of the stimulated muscle are achieved by train-of-four stimulation. ${ }^{35}$ Considering these potential drawbacks of neostigmine, its use for reversal is indicated when a patient's muscle strength has not recovered fully at the end of surgery, but the existence of residual neuromuscular blockade needs to be verified by objective, quantitative monitoring of neuromuscular transmission before the reversal agent can safely be administered.

\section{Strengths and weaknesses of the study}

The strength of this study is predominantly determined by the high quality database, which allowed us to focus on clinically meaningful endpoints while controlling for a variety of potential confounders. We created a propensity score matched cohort, which had a balanced risk factor and covariate profile reducing the possibility of confounding.

Several limitations should be considered when interpreting our results. The surgical procedure cases included in the registry are from a specialty medical center, which may limit generalizability to other settings. While we have no reason to believe that the effect of neuromuscular blocking agents on our outcomes was largely different in other centers, differences in managing perioperative muscle strength across centers may translate to different effects on respiratory outcome. This study of medical record data is observational in nature and residual and immeasurable confounding remains a possibility. In addition, we used the surgical time and surgical specialty carrying out the operations as proxies for the various procedures (as time of procedure and specialty are highly collinear with the individual procedures). In total, more than 5000 single categories were recorded, indicating various procedures. Adding this information to a multivariable model would not result in meaningful estimates. Finally, despite best efforts to gather complete and accurate data for each patient, it is not possible to rule out potential misclassification. This should, however, be non-differential.

\section{Possible biological explanations}

A growing body of evidence from volunteer studies and outcome studies suggests that adverse respiratory effects of non-depolarizing neuromuscular blocking agents are probably due to their lingering effects after surgery. ${ }^{10-15}$ Residual neuromuscular blockade increases the vulnerability of the respiratory system in such a way that adverse subclinical signs and symptoms occur. ${ }^{12}{ }^{15}$ Residual neuromuscular blockade also increases the incidence of adverse respiratory events in the 
post-anesthesia care unit, such as jaw thrust requirement, and increases the length of stay in the unit, ${ }^{10}{ }^{13-15}$ even after use of intermediate acting non-depolarizing neuromuscular blocking agents. ${ }^{42}$ Researchers found that residual paralysis was present in $45 \%$ of the cases on arrival in the post-anesthesia care unit up to two hours after use of a single dose of an intermediate acting muscle relaxant. ${ }^{42}$ On the basis of our data we speculate that lingering effects of neuromuscular blocking agents increase the risk of respiratory vulnerable patients to develop a respiratory compromise, ${ }^{10}{ }^{12-15}$ leading to unplanned admission to an intensive care unit. In accordance, in our study the risk of severe respiratory complications was higher in patients undergoing short ( $<2$ hours) surgical procedures, where the time between use of neuromuscular blocking agents to facilitate intubation and extubation may not have been long enough. Most of the patients undergoing surgical procedures in our study were reintubated for pulmonary edema, pneumonia, atelectasis, and aspiration. Based on this information, we conclude that it is possible that residual paralysis on the first day after surgery may be a contributing factor to reintubation on a subsequent day. Partial paralysis affects the ability to protect the airway during swallowing and should thereby increase the propensity to aspirate or develop pneumonia. The proposed mechanism of pulmonary edema may be negative pressure pulmonary edema; paralytics can induce an upper airway obstruction in the absence of respiratory pump muscle dysfunction. ${ }^{43}$

\section{Meaning of the study: implications for clinicians}

In the United States, about 20 million patients each year receive general anesthesia for surgery, ${ }^{44}$ and more than 100000000 units of muscle relaxants are being used in the United States in the operating theatre, emergency departments, and on the intensive care units. This study shows that the use of modern intermediate acting non-depolarizing neuromuscular blocking agents may put patients at risk for postoperative respiratory complications. Reintubation after surgery increases mortality and healthcare costs, ${ }^{16}{ }^{17}$ thus highlighting the potential implications of our results in terms of both patient safety and healthcare economics. Assuming causality, our results indicate that $26 \%$ of desaturation less than $90 \%, 40 \%$ of desaturation less than $80 \%$, and $29 \%$ of reintubations would be attributable to intermediate acting non-depolarizing neuromuscular blocking agents.

The mortality of postsurgical patients with unplanned admission to an intensive care unit is high, with respiratory complications being the most important problem. ${ }^{45}$ In our data, patients who needed reintubation requiring unplanned admission to an intensive care unit had a more than 90-fold higher risk of dying in the hospital (odds ratio 91, 95\% confidence interval 67 to 125) than patients who did not require intubation. In addition, critical care medicine is expensive. In the United States, treatment of critically ill patients represents nearly $1 \%$ of the gross domestic product and $25 \%$ of a typical hospital budget. Therefore, all efforts should be undertaken to reduce factors that contribute to unplanned admissions to an intensive care unit.

Desaturation after extubation may be influenced by many factors, such as the lingering effects of anesthetics, opioids, or neuromuscular blocking agents; the surgical trauma; or increased oxygen consumption from pain or sepsis. In addition, oxygen levels before extubation and the technique for extubation are likely important contributors. Our data show that the intraoperative use of intermediate acting non-depolarizing neuromuscular blocking agents, independent of common risk factors of respiratory outcome, is associated with severe postoperative pulmonary complications. Therefore, efforts should be made to optimize the use of neuromuscular blocking agents that have been shown to improve outcomes under well defined conditions. ${ }^{8}$

\section{Unanswered questions and future research}

Our data raise concern about the potential adverse outcome events of non-depolarizing neuromuscular blocking agents, and further targeted research is warranted to resolve remaining questions. For example, it is unclear if the respiratory morbidity associated with neuromuscular blocking agents can be mitigated by optimal clinical management such as the use of reversal agents and qualitative or quantitative monitoring of neuromuscular transmission or use of newer reversal agents such as sugammadex. ${ }^{33}{ }^{46}$ In summary, we found that use of intermediate acting neuromuscular blocking agents during anesthesia leads to an increased risk of developing clinically meaningful respiratory complications.

Contributors: MG-S carried out the data analysis and wrote substantial parts of the manuscript. She presented the study at the ASA annual meeting. JPH acquired the data and was involved in data analysis and writing the manuscript. MG-S and JPH contributed equally to the study. EM and BTB were involved in data analysis and revising the manuscript. JV-U was involved in data acquisition, analysis, and revising the manuscript. NTN was involved in data acquisition and revising the manuscript. JE was involved in designing the study, data acquisition, analysis, and writing. WS was involved in designing the study, data analysis, and revising the manuscript. TK was involved in designing the study, data analysis and interpretation, and writing. All authors had access to the data. ME was involved in all parts of the study and is the guarantor.

Funding: This study was funded by the Department of Anesthesia, Critical Care and Pain Medicine, Massachusetts General Hospital, Boston, United States.

Competing interests: All authors have completed the ICMJE uniform disclosure form at www.icmje.org/coi_disclosure.pdf (available on request from the corresponding author) and declare: that this study has been funded only by academic research funds; TK has received investigator initiated research funding from the French National Research Agency, the US National Institutes of Health, the Migraine Research Foundation, and the Parkinson's Disease Foundation. He has received honorariums from Allergan, the American Academy of Neurology, and Merck for educational lectures, from the $B M J$ for editorial services, and from MAP Pharmaceutical for contributing to a scientific advisory panel. $M E$ has received investigator initiated research funding from Merck, Pfizer, and the ResMed Foundation, as well as the Department of Anesthesia and Critical Care and Pain Medicine of the Massachusetts General Hospital. He has received honorariums from Hill-Rom for giving advise, and from the American Thoracic Society, Brown University, Michigan University, and Vanderbilt University for educational lectures, and from the Journal Anesthesiology for editorial services; the authors have no financial relationships with any organisation or company that might have an interest in the submitted work in the previous three years; and no other relationships or activities that could appear to have influenced the submitted work.

Ethical approval: This study was approved by Partners' human research committee in Boston, Massachusetts, USA (protocol No 2011P001300). Data sharing: No additional data available.

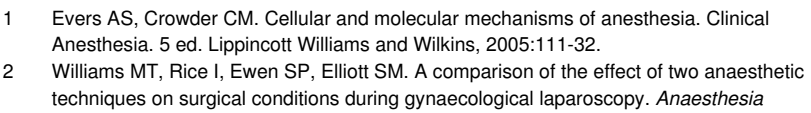




\section{What is already known on this topic}

Modern non-depolarizing neuromuscular blocking agents have a high market volume and are often used during surgery and on the intensive care unit

Long acting agents increase a patient's risk of postoperative respiratory complications, which may be related to residual neuromuscular blockade

Unanticipated reintubation after surgery is associated with increased mortality and increased healthcare costs

\section{What this study adds}

Use of intermediate acting non-depolarizing neuromuscular blocking agents during surgery are associated with an increased risk of severe postoperative, respiratory complications

Reversal of neuromuscular blockade using an acetylcholinesterase inhibitor at the end of surgery may increase a patient's risk of developing clinically meaningful respiratory complications.

Strategies to prevent postoperative residual neuromuscular blockade need to be revisited

3 Alfille PH, Merritt C, Chamberlin NL, Eikermann M. Control of perioperative muscle strength during ambulatory surgery. Curr Opin Anaesthesiol 2009;22:730-7.

4 Papazian L, Forel JM, Gacouin A, Penot-Ragon C, Perrin G, Loundou A, et al. Neuromuscular blockers in early acute respiratory distress syndrome. N Engl J Med 2010;363:1107-16

5 Eikermann M, Hunkemoller I, Peine L, Armbruster W, Stegen B, Husing J, et al. Optima rocuronium dose for intubation during inhalation induction with sevoflurane in children. Br J Anaesth 2002;89:277-81.

6 Mencke T, Echternach M, Kleinschmidt S, Lux P, Barth V, Plinkert PK, et al. Laryngeal morbidity and quality of tracheal intubation: a randomized controlled trial. Anesthesiology 2003;98:1049-56.

7 Mencke T, Echternach M, Plinkert PK, Johann U, Afan N, Rensing H, et al. Does the timing of tracheal intubation based on neuromuscular monitoring decrease laryngeal injury? A randomized, prospective, controlled trial. Anesth Analg 2006;102:306-12.

8 Wilcox SR, Bittner EA, Elmer J, Seigel TA, Nguyen NT, Dhillon A, et al. Neuromuscular blocking agent administration for emergent tracheal intubation is associated with decreased prevalence of procedure-related complications. Crit Care Med 2012;40:1808-13.

9 Segredo V, Caldwell JE, Matthay MA, Sharma ML, Gruenke LD, Miller RD. Persistent paralysis in critically ill patients after long-term administration of vecuronium. $N$ Engl $J$ Med 1992;327:524-8.

10 Butterly A, Bittner EA, George E, Sandberg WS, Eikermann M, Schmidt U. Postoperative residual curarization from intermediate-acting neuromuscular blocking agents delays recovery room discharge. Br J Anaesth 2010;105:304-9.

11 Eikermann M, Groeben H, Bunten B, Peters J. Fade of pulmonary function during residual neuromuscular blockade. Chest 2005:127:1703-9.

12 Eikermann M, Vogt FM, Herbstreit F, Vahid-Dastgerdi M, Zenge MO, Ochterbeck C, et al. The predisposition to inspiratory upper airway collapse during partial neuromuscular blockade. Am J Respir Crit Care Med 2007;175:9-15.

13 Murphy GS. Residual neuromuscular blockade: incidence, assessment, and relevance in the postoperative period. Minerva Anestesiol 2006;72:97-109.

14 Murphy GS, Szokol JW, Marymont JH, Greenberg SB, Avram MJ, Vender JS. Residual neuromuscular blockade and critical respiratory events in the postanesthesia care unit. Anesth Analg 2008;107:130-7.

15 Sundman E, Witt H, Olsson R, Ekberg O, Kuylenstierna R, Eriksson LI. The incidence and mechanisms of pharyngeal and upper esophageal dysfunction in partially paralyzed humans: pharyngeal videoradiography and simultaneous manometry after atracurium. Anesthesiology 2000:92:977-84.

16 Johnson RG, Arozullah AM, Neumayer L, Henderson WG, Hosokawa P, Khuri SF. Multivariable predictors of postoperative respiratory failure after general and vascular surgery: results from the patient safety in surgery study. J Am Coll Surg 2007;204:1188-98.

17 Ramachandran SK, Nafiu OO, Ghaferi A, Tremper KK, Shanks A, Kheterpal S. Independent predictors and outcomes of unanticipated early postoperative tracheal intubation after nonemergent, noncardiac surgery. Anesthesiology 2011:115:44-53.

18 Berg H, Roed J, Viby-Mogensen J, Mortensen CR, Engbaek J, Skovgaard LT, et at Residual neuromuscular block is a risk factor for postoperative pulmonary complications. A prospective, randomised, and blinded study of postoperative pulmonary complications after atracurium, vecuronium and pancuronium. Acta Anaesthesiol Scand 1997;41:1095-103.

19 Pedersen T, Viby-Mogensen J, Ringsted C. Anaesthetic practice and postoperative pulmonary complications. Acta Anaesthesiol Scand 1992;36:812-8.

20 Murphy GS, Brull SJ. Residual neuromuscular block: lessons unlearned. Part I: definitions, incidence, and adverse physiologic effects of residual neuromuscular block. Anesth Analg 2010;111:120-8.

21 Herbstreit F, Zigrahn D, Ochterbeck C, Peters J, Eikermann M. Neostigmine/glycopyrrolate administered after recovery from neuromuscular block increases upper airway collapsibility by decreasing genioglossus muscle activity in response to negative pharyngeal pressure. Anesthesiology 2010;113:1280-8.

22 Srivastava A, Hunter JM. Reversal of neuromuscular block. Br J Anaesth 2009;103:115-29.

23 Charlson ME, Pompei P, Ales KL, MacKenzie CR. A new method of classifying prognostic comorbidity in longitudinal studies: development and validation. J Chronic Dis 1987;40:373-83.

24 Parsons $\mathrm{L}$. Reducing bias in a propensity score matched-pair sample using greedy matching techniques. SAS Institute Proceedings of the 26th Annual SAS Users Group International Conference.

25 Kurth T, Walker AM, Glynn RJ, Chan KA, Gaziano JM, Berger K, et al. Results of multivariable logistic regression, propensity matching, propensity adjustment, and propensity-based weighting under conditions of nonuniform effect. Am J Epidemiol 2006;163:262-70.
26 Beecher HK, Todd DP. A study of the deaths associated with anesthesia and surgery: based on a study of 599, 548 anesthesias in ten institutions 1948-1952, inclusive. Ann Surg 1954;140:2-35.

27 Abajian J, Arrowood JG, Barrett RH, Dwyer CS, Eversole UH, Fine JH, et al. Critique of "A study of the deaths associated with anesthesia and surgery". Ann Surg 1955;142:138-41.

28 Harrison GG. Death attributable to anaesthesia. A 10-year survey (1967-1976). Br J Anaesth 1978;50:1041-6.

29 Lunn JN, Hunter AR, Scott DB. Anaesthesia-related surgical mortality. Anaesthesia 1983;38:1090-6.

30 Sprung J, Warner ME, Contreras MG, Schroeder DR, Beighley CM, Wilson GA, et al. Predictors of survival following cardiac arrest in patients undergoing noncardiac surgery: a study of 518,294 patients at a tertiary referral center. Anesthesiology 2003.99.259-69.

31 Arbous MS, Meursing AE, van Kleef JW, de Lange JJ, Spoormans HH, Touw P, et al. Impact of anesthesia management characteristics on severe morbidity and mortality. Anesthesiology 2005;102:257-68; quiz 491-2.

32 Rose DK, Cohen MM, Wigglesworth DF, DeBoer DP. Critical respiratory events in the postanesthesia care unit. Patient, surgical, and anesthetic factors. Anesthesiology 1994;81:410-8.

33 Murphy GS, Szokol JW, Marymont JH, Greenberg SB, Avram MJ, Vender JS, et al. Intraoperative acceleromyographic monitoring reduces the risk of residual neuromuscular blockade and adverse respiratory events in the postanesthesia care unit. Anesthesiology 2008;109:389-98.

34 Claudius C, Viby-Mogensen J. Acceleromyography for use in scientific and clinical practice: a systematic review of the evidence. Anesthesiology 2008;108:1117-40.

35 Kopman AF, Eikermann M. Antagonism of non-depolarising neuromuscular block: current practice. Anaesthesia 2009;64(Suppl 1):22-30.

36 Hayes AH, Mirakhur RK, Breslin DS, Reid JE, McCourt KC. Postoperative residual block after intermediate-acting neuromuscular blocking drugs. Anaesthesia 2001;56:312-8.

37 Payne JP, Hughes R, Al Azawi S. Neuromuscular blockade by neostigmine in anaesthetized man. Br J Anaesth 1980;52:69-76.

38 Caldwell JE. Reversal of residual neuromuscular block with neostigmine at one to four hours after a single intubating dose of vecuronium. Anesth Analg 1995;80:1168-74.

39 Eikermann M, Fassbender P, Malhotra A, Takahashi M, Kubo S, Jordan AS, et al. Unwarranted administration of acetylcholinesterase inhibitors can impair genioglossus and diaphragm muscle function. Anesthesiology 2007;107:621-9.

40 Paton F, Paulden M, Chambers D, Heirs M, Duffy S, Hunter JM, et al. Sugammadex compared with neostigmine/glycopyrrolate for routine reversal of neuromuscular block: a systematic review and economic evaluation. Br J Anaesth 2010;105:558-67.

41 Beemer GH, Bjorksten AR, Dawson PJ, Dawson RJ, Heenan PJ, Robertson BA. Determinants of the reversal time of competitive neuromuscular block by anticholinesterases. Br J Anaesth 1991;66:469-75.

42 Debaene B, Plaud B, Dilly MP, Donati F. Residual paralysis in the PACU after a single intubating dose of nondepolarizing muscle relaxant with an intermediate duration of action. Anesthesiology 2003;98:1042-8.

43 Krodel DJ, Bittner EA, Abdulnour R, Brown R, Eikermann M. Case scenario: acute postoperative negative pressure pulmonary edema. Anesthesiology 2010;113:200-7.

44 Brown EN, Lydic R, Schiff ND. General anesthesia, sleep, and coma. N Engl J Med 2010;363:2638-50.

45 Chan KS, Tan CK, Fang CS, Tsai CL, Hou CC, Cheng KC, et al. Readmission to the intensive care unit: an indicator that reflects the potential risks of morbidity and mortality of surgical patients in the intensive care unit. Surg Today 2009;39:295-9.

46 Eikermann M, Zaremba S, Malhotra A, Jordan AS, Rosow C, Chamberlin NL. Neostigmine but not sugammadex impairs upper airway dilator muscle activity and breathing. $\mathrm{Br} \mathrm{J}$ Anaesth 2008;101:344-9.

Accepted: 11 September 2012

\section{Cite this as: BMJ 2012;345:e6329}

This is an open-access article distributed under the terms of the Creative Commons Attribution Non-commercial License, which permits use, distribution, and reproduction in any medium, provided the original work is properly cited, the use is non commercial and is otherwise in compliance with the license. See: http://creativecommons.org/licenses/by$\mathrm{nc} / 2.0 /$ and http://creativecommons.org/licenses/by-nc/2.0/legalcode. 


\section{Tables}

Table 1| Characteristics of study population according to whether or not intermediate acting non-depolarizing neuromuscular blocking agents were administered intraoperatively. Values are frequencies (percentages) unless stated otherwise

\begin{tabular}{|c|c|c|}
\hline \multirow[b]{2}{*}{ Subgroups } & \multicolumn{2}{|c|}{ Neuromuscular blocking agents } \\
\hline & Administered $(n=32$ 002) & Not administered $(n=25066)$ \\
\hline \multicolumn{3}{|l|}{ Age (years): } \\
\hline$<10$ & $966(3.0)$ & $1495(6.0)$ \\
\hline $10-19$ & $1383(4.3)$ & $1415(5.7)$ \\
\hline $20-29$ & $2362(7.4)$ & $1874(7.5)$ \\
\hline $30-39$ & $3276(10.3)$ & $2547(10.2)$ \\
\hline $40-49$ & $5102(16.0)$ & $4424(17.7)$ \\
\hline $50-59$ & $6154(19.2)$ & $4872(19.4)$ \\
\hline $60-69$ & $5813(18.2)$ & $4258(17.0)$ \\
\hline $70-79$ & $3801(11.9)$ & $2360(9.4)$ \\
\hline$\geq 80$ & $1821(5.7)$ & $936(3.7)$ \\
\hline Missing & $1324(4.1)$ & $885(3.5)$ \\
\hline Mean (SD) & $51.2(19.8)$ & $47.6(20.7)$ \\
\hline Female & $17294(54.0)$ & $13054(52.1)$ \\
\hline Male & $14708(46.0)$ & $12012(47.9)$ \\
\hline \multicolumn{3}{|c|}{ Body weight fifths $(\mathrm{kg})$ : } \\
\hline$<60$ & 5504 (17.2) & $4710(18.8)$ \\
\hline $60-69.9$ & $5664(17.7)$ & $4332(17.3)$ \\
\hline $70-80.9$ & $6026(18.8)$ & $4510(18.0)$ \\
\hline $81-94.9$ & $5783(18.1)$ & 4419 (17.6) \\
\hline$\geq 95$ & $5974(18.7)$ & $4315(19.9)$ \\
\hline Missing & 3051 (9.5) & $2780(11.1)$ \\
\hline Mean (SD) & $77.8(24.6)$ & $75.9(26.6)$ \\
\hline \multicolumn{3}{|l|}{ ASA status: } \\
\hline 1 & 4581 (14.3) & $5201(20.8)$ \\
\hline 2 & $18330(57.3)$ & $14568(58.1)$ \\
\hline 3 & $8165(25.5)$ & $4645(18.5)$ \\
\hline$\geq 4$ & $446(1.4)$ & $222(0.9)$ \\
\hline Missing & $480(1.5)$ & $430(1.7)$ \\
\hline Mean (SD) & $2.1(0.7)$ & $2.0(0.7)$ \\
\hline \multicolumn{3}{|c|}{ Charlson comorbidity index: } \\
\hline 0 & $15597(48.7)$ & $15152(60.5)$ \\
\hline 1 & $5742(17.9)$ & 3329 (13.3) \\
\hline 2 & $2948(9.2)$ & $1848(7.4$ \\
\hline 3 & $3940(12.3)$ & $2662(10.2)$ \\
\hline 4 & $1934(6.0)$ & $1062(4.2)$ \\
\hline 5 & $689(2.2)$ & $360(1.4)$ \\
\hline$\geq 6$ & $1152(3.6)$ & $653(2.6)$ \\
\hline Mean (SD) & $1.1(2.1)$ & $1.4(2.3)$ \\
\hline \multicolumn{3}{|l|}{ Surgical specialty: } \\
\hline Burn & $373(1.2)$ & $248(1.0)$ \\
\hline Cardiac surgery & $86(0.3)$ & $18(0.1)$ \\
\hline General surgery & $5337(16.7)$ & $2758(11.0)$ \\
\hline Gynecology & $2642(8.3)$ & $2128(8.5)$ \\
\hline Neurosurgery & 3062 (9.6) & $1219(4.9)$ \\
\hline
\end{tabular}


Table 1 (continued)

\begin{tabular}{|c|c|c|}
\hline \multirow[b]{2}{*}{ Subgroups } & \multicolumn{2}{|c|}{ Neuromuscular blocking agents } \\
\hline & Administered ( $n=32$ 002) & Not administered $(n=25066)$ \\
\hline Oral/maxillofacial surgery & $959(3.0)$ & $349(1.4)$ \\
\hline Orthopedic surgery & $6094(19.0)$ & $6355(25.4)$ \\
\hline Pediatric surgery & $1250(3.9)$ & $1684(6.7)$ \\
\hline Plastic surgery & $1599(5.0)$ & $953(3.8)$ \\
\hline Surgical oncology & $1336(4.2)$ & $2590(10.3)$ \\
\hline Thoracic surgery & $3118(9.7)$ & $1418(5.7)$ \\
\hline Transplant surgery & $548(1.7)$ & $384(1.5)$ \\
\hline Trauma surgery & $1837(5.7)$ & $630(2.5)$ \\
\hline Urology & $1998(6.2)$ & $3377(13.5)$ \\
\hline Vascular surgery & $1376(4.3)$ & $479(1.9)$ \\
\hline Other & $45(0.1)$ & $226(0.9)$ \\
\hline Missing & $342(1.1)$ & $250(1.0)$ \\
\hline \multicolumn{3}{|l|}{ Duration of surgery $(\mathrm{min})$ : } \\
\hline$<120$ & $20432(64.1)$ & $18774(76.3)$ \\
\hline$\geq 120$ & $11445(35.9)$ & $5846(23.7)$ \\
\hline Missing & $125(0.4)$ & $446(1.8)$ \\
\hline Mean (SD) & $111.3(92.8)$ & $86.8(94.4)$ \\
\hline Emergency case & $1698(5.3)$ & $734(2.9)$ \\
\hline Missing & $480(1.5)$ & $430(1.7)$ \\
\hline \multicolumn{3}{|l|}{ Volatile anesthetics: } \\
\hline Any & $29764(93.0)$ & $22841(91.1)$ \\
\hline Desflurane & $1639(5.1)$ & $1437(5.7)$ \\
\hline Isoflurane & $10003(31.3)$ & $3451(13.8)$ \\
\hline Sevoflurane & $20459(63.9)$ & $19066(76.1)$ \\
\hline Nitrous oxide & $26801(83.8)$ & $21401(85.4)$ \\
\hline \multicolumn{3}{|l|}{ Strategies to avoid residual neuromuscular blockade: } \\
\hline Neostigmine & $20351(63.6)$ & NA \\
\hline Neuromuscular transmission monitoring & $15997(50.0)$ & NA \\
\hline Neostigmine plus neuromuscular transmission monitoring & $11905(37.2)$ & NA \\
\hline
\end{tabular}

ASA=American Society of Anesthesiologists; NA=not applicable. Percentages may not add up to $100 \%$ because of rounding. 
Table 2| Characteristics of cases in propensity score matched cohort $(n=37$ 158) of patients who did or did not receive at least one intermediate acting non-depolarizing neuromuscular blocking agent. Values are frequencies (percentages) unless stated otherwise

\begin{tabular}{|c|c|c|}
\hline \multirow[b]{2}{*}{ Subgroups } & \multicolumn{2}{|c|}{ Neuromuscular blocking agents } \\
\hline & Received ( $n=18$ 579) & Not received $(n=18579)$ \\
\hline \multicolumn{3}{|l|}{ Age (years): } \\
\hline $1-9$ & $795(4.3)$ & $733(4.0)$ \\
\hline $10-19$ & $958(5.2)$ & $938(5.1)$ \\
\hline $20-29$ & $1446(7.8)$ & $1419(7.6)$ \\
\hline 30-39 & $1951(10.5)$ & $1971(10.6)$ \\
\hline $40-49$ & $3193(17.2)$ & $3220(17.3)$ \\
\hline $50-59$ & $3707(20.0)$ & $3738(20.1)$ \\
\hline $60-69$ & $3252(17.5)$ & $3268(17.6)$ \\
\hline $70-79$ & $1840(9.9)$ & $1831(9.9)$ \\
\hline$\geq 80$ & $750(4.0)$ & $759(4.1)$ \\
\hline Missing & $687(3.7)$ & $702(3.8)$ \\
\hline Mean (SD) & $48.9(20.0)$ & $49.1(19.8)$ \\
\hline Female & $9638(51.9)$ & $9535(51.3)$ \\
\hline Male & $8941(48.1)$ & $9044(48.7)$ \\
\hline \multicolumn{3}{|l|}{ Body weight (kg): } \\
\hline$<60$ & $3265(17.6)$ & $3150(17.0)$ \\
\hline $60-69.9$ & $3225(17.4)$ & $3217(17.3)$ \\
\hline $70-80.9$ & $3416(18.4)$ & $3441(18.5)$ \\
\hline $81-94.9$ & $3259(17.5)$ & $3386(18.2)$ \\
\hline$\geq 95$ & 3579 (19.3) & $3526(19.0)$ \\
\hline Missing & $1835(9.9)$ & $1859(10.0)$ \\
\hline Mean (SD) & $77.5(25.8)$ & $77.1(25.8)$ \\
\hline \multicolumn{3}{|l|}{ ASA score: } \\
\hline 1 & $3230(17.4)$ & $3182(17.1)$ \\
\hline 2 & $10892(58.6)$ & $10905(58.7)$ \\
\hline 3 & $3965(21.3)$ & $3989(21.5)$ \\
\hline$\geq 4$ & $202(1.1)$ & $196(1.1)$ \\
\hline Missing & $290(1.6)$ & $307(1.7)$ \\
\hline Mean (SD) & $2.1(0.7)$ & $2.1(0.7)$ \\
\hline \multicolumn{3}{|l|}{ Charlson comorbidity index: } \\
\hline 0 & $10069(54.2)$ & $10083(54.3)$ \\
\hline 1 & $2945(15.9)$ & $2891(15.6)$ \\
\hline 2 & $1566(8.4)$ & $1555(8.4)$ \\
\hline 3 & $2212(11.9)$ & $2248(12.1)$ \\
\hline 4 & $940(5.1)$ & $922(5.0)$ \\
\hline 5 & $312(1.7)$ & $329(1.8)$ \\
\hline$\geq 6$ & $535(2.9)$ & $551(3.0)$ \\
\hline Mean (SD) & $1.3(2.2)$ & $1.3(2.2)$ \\
\hline \multicolumn{3}{|l|}{ Surgical specialty: } \\
\hline Burn & $217(1.2)$ & $235(1.3)$ \\
\hline Cardiac surgery & $26(0.1)$ & $18(0.1)$ \\
\hline General surgery & $2783(15.0)$ & $2624(14.1)$ \\
\hline Gynecology & $1486(8.0)$ & $1581(8.5)$ \\
\hline Neurosurgery & $1267(6.8)$ & $1200(6.5)$ \\
\hline Oral/maxillofacial surgery & $356(1.9)$ & 347 (1.9) \\
\hline Orthopedic surgery & $4397(23.7)$ & $4546(24.5)$ \\
\hline Pediatric surgery & $974(5.2)$ & $921(5.0)$ \\
\hline
\end{tabular}


Table 2 (continued)

\begin{tabular}{|c|c|c|}
\hline \multirow[b]{2}{*}{ Subgroups } & \multicolumn{2}{|c|}{ Neuromuscular blocking agents } \\
\hline & Received ( $n=18$ 579) & Not received $(n=18579)$ \\
\hline Plastic surgery & $823(4.4)$ & $876(4.7)$ \\
\hline Surgical oncology & $1317(7.1)$ & $1198(6.5)$ \\
\hline Thoracic surgery & $1320(7.1)$ & $1391(7.5)$ \\
\hline Transplant surgery & $328(1.8)$ & $353(1.9)$ \\
\hline Trauma surgery & $641(3.5)$ & $624(3.4)$ \\
\hline Urology & $1924(10.4)$ & $1967(10.6)$ \\
\hline Vascular surgery & $479(2.6)$ & $470(2.5)$ \\
\hline Other & $45(0.2)$ & $26(0.1)$ \\
\hline Missing & $196(1.1)$ & $202(1.1)$ \\
\hline \multicolumn{3}{|l|}{ Duration of surgery $(\mathrm{min})$ : } \\
\hline$<120$ & $12609(67.9)$ & $12836(69.1)$ \\
\hline$\geq 120$ & $5845(31.5)$ & $5652(30.4)$ \\
\hline Missing & $125(0.7)$ & $91(0.5)$ \\
\hline Mean (SD) & $100.1(95.4)$ & $102.6(101.5)$ \\
\hline Emergency case & $635(3.4)$ & $672(3.6)$ \\
\hline Missing & $290(1.6)$ & $307(1.7)$ \\
\hline Volatile anesthetics & $17134(92.2)$ & $17103(92.1)$ \\
\hline Nitrous oxide & $15803(85.1)$ & $15729(84.7)$ \\
\hline \multicolumn{3}{|l|}{ Strategies to avoid residual neuromuscular blockade: } \\
\hline Neostigmine & $11737(63.2)$ & NA \\
\hline Neuromuscular transmission monitoring & $9081(48.9)$ & NA \\
\hline Neostigmine plus neuromuscular transmission monitoring & $6732(36.2)$ & NA \\
\hline
\end{tabular}

ASA=American Society of Anesthesiologists; NA=not applicable. Percentages may not add up to $100 \%$ because of rounding. 
Table 3| Association between use of intermediate acting non-depolarizing neuromuscular blocking agents and outcomes in propensity score matched cohort $(n=37$ 158)

Neuromuscular blocking agents

Outcomes Not received $(n=18579)$ Received $(n=18579)$ Odds ratio $(95 \% \mathrm{Cl})$

\begin{tabular}{lccc} 
Desaturation $<90 \%$ & 689 & 925 & $1.36(1.23$ to 1.51$)$ \\
\hline Desaturation $<80 \%$ & 128 & 212 & 1.66 (1.34 to 2.07$)$ \\
\hline Reintubation & 108 & 151 & 1.40 (1.09 to 1.80$)$ \\
\hline In-hospital death & 48 & 55 & 1.15 (0.78 to 1.69$)$ \\
\hline
\end{tabular}


Table 4| Association between strategies to prevent partial neuromuscular blockade after surgery (neostigmine reversal and neuromuscular transmission monitoring) and outcomes in propensity score matched cohort ( $n=37$ 158)

\begin{tabular}{|c|c|c|c|c|c|c|}
\hline \multirow[b]{2}{*}{ Outcomes } & \multicolumn{2}{|c|}{ Neostigmine } & \multirow[b]{2}{*}{ Odds ratio $(95 \% \mathrm{Cl})$} & \multicolumn{2}{|c|}{ Neuromuscular transmission monitoring } & \multirow[t]{2}{*}{ Odds ratio $(95 \% \mathrm{Cl})$} \\
\hline & $\begin{array}{l}\text { Not administered } \\
\quad(n=21 \text { 117) }\end{array}$ & $\begin{array}{l}\text { Administered }(\mathrm{n}=16 \\
041)\end{array}$ & & Not applied ( $n=24281)$ & Applied ( $n=12$ 877) & \\
\hline Desaturation $<90 \%$ & 810 & 804 & $1.32(1.20$ to 1.46$)$ & 993 & 621 & 1.19 (1.07 to 1.32$)$ \\
\hline Desaturation $<80 \%$ & 180 & 160 & $1.17(0.95$ to 1.45$)$ & 211 & 129 & $1.15(0.93$ to 1.44$)$ \\
\hline Reintubation & 111 & 148 & 1.76 (1.38 to 2.26$)$ & 145 & 114 & $1.49(1.16$ to 1.90$)$ \\
\hline In-hospital death & 58 & 45 & 1.02 (0.69 to 1.51$)$ & 66 & 37 & 1.06 (0.71 to 1.58$)$ \\
\hline
\end{tabular}




\section{Figures}

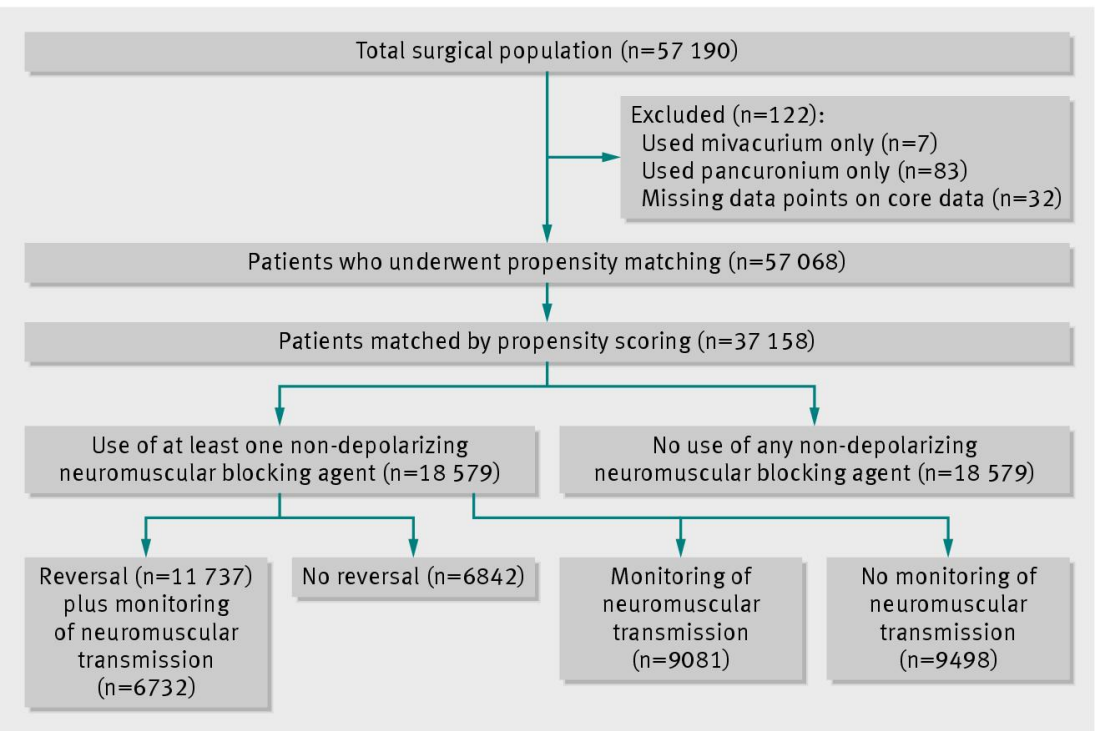

Fig 1 Study design

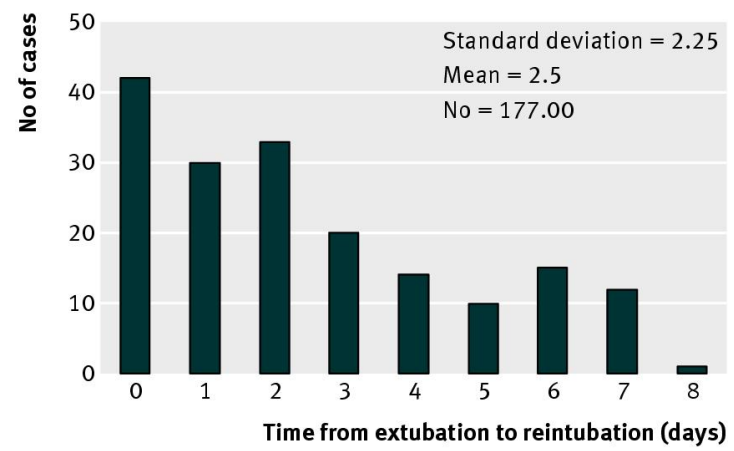

Fig 2 Duration of mechanical ventilation after reintubation based on chart review

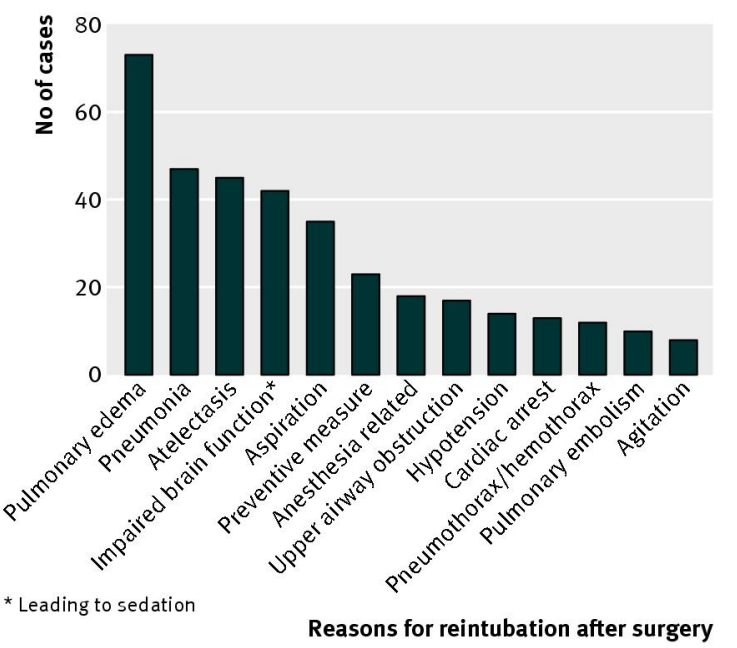

Fig 3 Reasons for reintubation in patients undergoing surgical procedures and reintubated after surgery in post-anesthesia care unit, on the surgical floor, or on the intensive care unit. Data taken from chart review. Data given from patients only with clear documentation of cause and effect relation between mechanism of respiratory disease and intubation ( $n=177)$. Multiple reasons (on average 2.5 per reintubation) were provided 